\title{
Perceptual Color Correction: A Variational Perspective
}

\author{
Edoardo Provenzi \\ Departament de Tecnologies de la Informació i les Comunicacions, Universitat \\ Pompeu Fabra, C/Tànger 122-140, 08018, Barcelona, Spain
}

\begin{abstract}
Variational techniques provide a powerful tool for understanding image features and creating new efficient algorithms. In the past twenty years, this machinery has been also applied to color images. Recently, a general variational framework that incorporates the basic phenomenological characteristics of the human visual system has been built. Here we recall the structure of this framework and give noticeable examples. We then propose a new analytic expression for a parameter that regulates contrast enhancement. This formula is defined in terms of intrinsic image features, so that the parameter no longer needs to be empirically set by a user, but it is automatically determined by the image itself.
\end{abstract}

\section{Introduction}

Human vision is a process of great complexity that involves many features as, e.g. shape and pattern recognition, movement analysis and color perception. In this paper we will focus on this last one. This process begins with light capture by the three different types of cone pigments inside the retina. When a light stimulus activates a cone, a photochemical transition occurs, producing a nerve impulse that reaches the brain, where it is analyzed and interpreted. However, neither retina photochemistry nor nervous impulses propagation are well understood, hence a deterministic characterization of the visual process is unavailable. For this reason, the majority of color perception models follow a descriptive approach, trying to simulate macroscopic characteristics of color vision, rather than reproduce neurophysiological activity.

A common characteristic of these models is that the Human Visual System (HVS) features are taken as inspiration to devise the explicit equations of a perceptual algorithms. The point of view adopted in [1] is rather different, since it focuses on the translation of the basic macroscopic phenomenological characteristics of color vision into mathematical axioms to be fulfilled by a variational energy functional in order to be considered perceptually inspired. Remarkably, it can be proven that only one class of energy functionals can cumply with all the axioms at once.

Once a perceptual energy is fixed, the Euler-Lagrange equations corresponding to its minimization give rise to a computational algorithm that can be used to perform perceptual color correction. The advantage of this point of view

A. Trémeau, R. Schettini, and S. Tominaga (Eds.): CCIW 2009, LNCS 5646, pp. 109-119, 2009.

(C) Springer-Verlag Berlin Heidelberg 2009 
relies in the intertwining between the algorithm equation and the corresponding variational energy, which permits to better understand the algorithm behavior in terms of important image features as tone dispersion or contrast.

A parameter appearing in these models is a coefficient which controls the degree of contrast enhancement. Here we propose an image-driven formula that respects the HVS phenomenology and automatically set this parameter pixel-wise, so that it does not need to be superimposed by a user.

\section{Basic Human Color Perception Phenomenology}

The phenomenological properties of human color perception that we consider as basics for our axiomatic construction are three: color constancy, local contrast enhancement and visual adaptation.

Let us begin with human color constancy: It is well known that the HVS has strong 'normalization' properties, i.e. humans can perceive colors of a scene almost independently of the spectral electromagnetic composition of a uniform illuminant, usually called color cast [2]. This peculiar ability is known as human color constancy and it is not perfect [3] because, even though human color perception strongly depends on the context, i.e. on the relative radiance rather than on the absolute one, also absolute luminance information plays a role in the entire color perception process.

The majority of color modeFor this reason, the majority of color perception models follow a descriptive approach, trying to simulate macroscopic characteristics of color vision, rather than reproduce neurophysiological activity.ls try to remove color cast due to illuminant looking for the invariant component of the light signal: the physical reflectance of objects. However it is well known that the separation between illuminant and reflectance is an ill-posed problem, unless one imposes further constraints which are not verified by all images [4. Instead, in [1, another approach based on contrast enhancement is used to remove color cast. That method is based on the consideration that an image with color cast is always characterized by one chromatic channel with remarkably different standard deviation $\sigma$ with respect to the others (not to be confused with an image with dominant color, e.g. the close up of a leaf, in which the average value of a channel prevails). Since standard deviation is a measure of average contrast, it is clear that contrast enhancement can help decreasing the difference between $\sigma_{R}$, $\sigma_{G}$ and $\sigma_{B}$, by spreading the intensity values of all separate chromatic channels, thus reducing color cast.

Let us now recall the local contrast enhancement property: while looking at a natural scene, the HVS enhances local edges in order to better distinguish shapes and objects. Well known phenomena exhibiting this effect are Mach bands and simultaneous contrast [5]. Local HVS features related to color perception can be detected also in more complex scenes and go under the general name of 'chromatic induction' 677. Experimental evidences show that the strength of chromatic induction between two different areas of a scene decreases monotonically with their Euclidean distance, even though a precise analytical description is not yet available [7. 
Finally, let us remember the adaptation properties of the HVS. The range of light intensity levels to which the HVS can adapt is impressive, on the order of $10^{10}$, from the scotopic threshold to the glare limit [5]. However, the HVS system cannot operate over such a range simultaneously, rather it adapts to each scene to prevent saturation which would depress contrast sensitivity [8].

During this adaptation, the HVS shifts its sensitivity to light stimuli in order to present only modulations around the average intensity level of a scene 8 . This provides a phenomenological motivation for the so-called 'gray-world' (GW) assumption, which says that the average color in a scene is gray [9].

\section{Assumptions for a Perceptually-Inspired Variational Energy Functional}

The content of this section have been discussed and proved in detail in [1]. Here we only aim at summarizing the most relevant information of that work.

Let us fix the notation. Given a discrete RGB image, we denote by $\mathfrak{I}=$ $\{1, \ldots, W\} \times\{1, \ldots, H\} \subset \mathbb{Z}^{2}$ its spatial domain, $W, H \geq 1$ being integers; $x=\left(x_{1}, x_{2}\right)$ and $y=\left(y_{1}, y_{2}\right)$ denote the coordinates of two arbitrary pixels in $\mathfrak{I}$. We will always consider a normalized dynamic range in $[0,1]$, so that a color image function is denoted by $\boldsymbol{I}: \mathfrak{I} \rightarrow[0,1]^{3}, \boldsymbol{I}(x)=\left(I_{R}(x), I_{G}(x), I_{B}(x)\right)$, where $I_{c}(x)$ is the intensity level of the pixel $x \in \mathfrak{I}$ in the chromatic channel $c \in\{R, G, B\}$. All computations will be performed on the scalar components of the image, thus treating independently each chromatic channel, written, for simplicity, as $I(x)$.

\subsection{Assumption 1: General Structure of a Perceptual Energy Functional}

In order to write the general structure of a perceptually inspired energy functional, note that human color perception is characterized by both local and global features: contrast enhancement has a local nature, i.e. spatially variant, while visual adaptation and attachment to original data (implied by the failure of color constancy) have a global nature, i.e. spatially invariant, in the sense that they do not depend on the intensity distribution in the neighborhood.

These basic considerations imply that a perceptually inspired energy functional should contain two terms: one spatially-dependent term whose minimization leads to a local contrast enhancement and one global term whose minimization leads to a control of the departure from both original pointwise values and the middle gray, which, in our normalized dynamic range, is $1 / 2$.

Let us first describe the general form of the contrast enhancement terms. For that we need a contrast measure $\bar{c}(a, b)$ between two gray levels $a, b>0$ (to avoid some singular cases, we shall assume that intensity image values are always positive). We require the contrast function $\bar{c}:(0,+\infty) \times(0,+\infty) \rightarrow \mathbb{R}$ to be continuous, symmetric in $(a, b)$, i.e. $\bar{c}(a, b)=\bar{c}(b, a)$, increasing when $\min (a, b)$ decreases or $\max (a, b)$ increases. Basic examples of contrast measures are $\bar{c}=$ $|a-b| \equiv \max (a, b)-\min (a, b)$ or $\bar{c}(a, b)=\frac{\max (a, b)}{\min (a, b)}$. 
Since our purpose is to enhance contrast by minimizing an energy, we define an inverse contrast function $c(a, b)$, still continuous and symmetric in $(a, b)$, but decreasing when $\min (a, b)$ decreases or $\max (a, b)$ increases. Notice that, if $\bar{c}(a, b)$ is a contrast measure, then $c(a, b)=-\bar{c}(a, b)$ or $c(a, b)=1 / \bar{c}(a, b)$ is an inverse contrast measure, so that basic examples of inverse contrast are: $c(a, b)=\min (a, b)-\max (a, b)$ or $c(a, b)=\frac{\min (a, b)}{\max (a, b)}$.

Let us now introduce a normalized weighting function to localize the contrast computation. Let $w: \mathfrak{I} \times \mathfrak{I} \rightarrow \mathbb{R}^{+}$be a positive symmetric kernel, i.e. such that $w(x, y)=w(y, x)>0$, for all $x, y \in \mathfrak{I}$, that measures the mutual influence between the pixels $x, y$. The symmetry requirement is motivated by the fact that the mutual chromatic induction is independent on the order of the two pixel considered. Usually, we assume that $w(x, y)$ is a function of the Euclidean distance $\|x-y\|_{\mathfrak{I}}$ between the two points. We shall assume that the kernel is normalized, i.e. that

$$
\sum_{y \in \mathfrak{I}} w(x, y)=1 \quad \forall x \in \mathfrak{I} .
$$

Given an inverse contrast function $c(a, b)$ and a positive symmetric kernel $w(x, y)$, we define a contrast energy term by

$$
C_{w}(I)=\sum_{x \in \mathfrak{I}} \sum_{y \in \mathfrak{I}} w(x, y) c(I(x), I(y)) .
$$

The symmetry assumption implies that $c(a, b)=\tilde{c}(\min (a, b), \max (a, b))$ for some function $\tilde{c}$ (indeed well defined by this identity). Notice that $\tilde{c}$ is non-decreasing in the first argument and non-increasing in the second one.

Let us now consider the term that should control the dispersion. As suggested previously, it should realize an attachment to the initial given image $I_{0}$ and to the average illumination value, which we assume to be $1 / 2$. Thus, we define two dispersion functions: $d_{1}\left(I(x), I_{0}(x)\right)$ to measure the separation between $I(x)$ and $I_{0}(x)$, and $d_{2}\left(I(x), \frac{1}{2}\right)$ which measures the separation from the value $1 / 2$. Both $d_{1}$ and $d_{2}$ are continuous functions $d_{1,2}: \mathbb{R}^{2} \rightarrow \mathbb{R}^{+}$such that $d_{1,2}(a, a)=0$ for any $a \in \mathbb{R}$, and $d_{1,2}(a, b)>0$ if $a \neq b$. We write $d_{I_{0}, \frac{1}{2}}(I(x))=d_{1}\left(I(x), I_{0}(x)\right)+$ $d_{2}\left(I(x), \frac{1}{2}\right)$, and the dispersion energy term as

$$
D(I)=\sum_{x \in \mathfrak{I}} d_{I_{0}, \frac{1}{2}}(I(x)) .
$$

We can now formulate our first assumption.

Assumption 1. The general structure of a perceptually inspired color correction energy functional is

$$
E_{w}(I)=D(I)+C_{w}(I),
$$

where $C_{w}(I)$ and $D(I)$ are the contrast and dispersion terms defined in (2) and (3), respectively. The minimization of D must provide a control of the dispersion around 1/2 and around the original intensity values. The minimization of $C_{w}$ must provide a local contrast enhancement. 


\subsection{Assumption 2: Properties of the Contrast Function}

In order to find out which properties the contrast term should satisfy, let us observe that an overall change in intensity, measured by the generic quantity $\lambda>0$, does not affect the visual sensation. This requires the contrast function $c$ to be homogeneous, recalling that $c$ is homogeneous of degree $n \in \mathbb{Z}$ if

$$
c(\lambda a, \lambda b)=\lambda^{n} c(a, b) \quad \forall \lambda, a, b \in(0,+\infty),
$$

where $a$ and $b$ are synthetic representations of $I(x)$ and $I(y)$. Of course, if $n=0$, $c$ automatically disregards the presence of $\lambda$, but we can say more: since $\lambda$ can take any positive value, if we set $\lambda=1 / b$, we may write equation (5) as:

$$
c(a, b)=b^{n} c\left(\frac{a}{b}, 1\right) \quad \forall a, b \in(0,+\infty),
$$

so, when $n=0, b^{n}=1$ and thus $c$ results as a function of the ratio $a / b$ which intrinsically disregards overall changes in light intensity. If $n>0$, then $\lambda$ has a global influence and could be removed performing a suitable normalization (for instance, dividing by the $n$-th power of the highest intensity level). We can formalize these considerations in our second assumption.

Assumption 2. We assume that the inverse contrast function $c(a, b)$ is homogeneous.

Thanks to the arguments presented so far, we have that inverse contrast functions which are homogeneous of degree $n=0$ are those that can be written as a monotone non-decreasing function of $\frac{\min (I(x), I(y))}{\max (I(x), I(y))}$.

Let us now introduce into the discussion the important Weber-Fechner's law [5] which says that the so-called Weber-Fechner ratio $\mathcal{R}_{W F} \equiv \frac{I_{1}-I_{0}}{I_{0}}$, i.e. the ratio between an intensity variation $\Delta I \equiv I_{1}-I_{0}$ and the background intensity $I_{0}$, remains constant. The consequence is that the same variation is perceived in a weaker way as the strenght of the intensity increases. Even though WeberFechner's law is not perfect [10, the intensity range over which it is in good agreement with experience (called 'Weber-Fechner's domain') is still comparable to the dynamic range of most electronic imaging systems [10].

Since $\mathcal{R}_{W F}=I_{1} / I_{0}-1$, Weber-Fechner's law is saying that the perceived contrast is a function of $I_{1} / I_{0}$. This reason motivates us to say that $c(a, b)$ is a generalized Weber-Fechner contrast function if $c$ is an inverse contrast function which can be written as a non-decreasing function of $\min (a, b) / \max (a, b)$. Hence, we can particularize assumption 2 as follows.

Assumption 2'. We assume that $c$ is a generalized Weber-Fechner contrast function.

Noticeable examples of contrast terms are the following:

$$
C_{w}^{\text {id }}(I):=\frac{1}{4} \sum_{x \in \mathcal{I}} \sum_{y \in \mathfrak{I}} w(x, y) \frac{\min (I(x), I(y))}{\max (I(x), I(y))},
$$




$$
C_{w}^{\log }(I):=\frac{1}{4} \sum_{x \in \mathfrak{I}} \sum_{y \in \mathfrak{I}} w(x, y) \log \left(\frac{\min (I(x), I(y))}{\max (I(x), I(y))}\right),
$$

The upper symbol in the above definitions of $C_{w}$ simply specifies the monotone function applied on the basic contrast variable $t:=\frac{\min (I(x), I(y))}{\max (I(x), I(y))}$. To refer to any one of them we use the notation $C_{w}^{f}(I)$, where $f=\mathrm{id}$, log. Notice that the function $t=\min (I(x), I(y)) / \max (I(x), I(y))$ is minimized when $\min (I(x), I(y))$ takes the smallest possible value and $\max (I(x), I(y))$ takes the largest possible one, which corresponds to a contrast stretching. Thus, minimizing an increasing function of the variable $t$, will produce a contrast enhancement.

\subsection{Assumption 3: Entropic Dispersion Term}

The main features of the dispersion term have to be its attachment to the initial given image $I_{0}$ and to the average illumination value, which we assume to be $1 / 2$. In principle, to measure the dispersion of $I$ with respect to $I_{0}$ or $1 / 2$, any distance function can be used. However, let us notice that, given that contrast terms are expressed as homogeneous functions of degree 0 , the variational derivatives are homogeneous functions of degree -1 . Since the previous axioms do not give any precise indication about the analytical form of the dispersion term that should be chosen, we search for functions able to maintain coherence with this homogeneity. A good candidate for this is the entropic dispersion term $D_{\alpha, \beta}^{\mathcal{E}}(I)$ :

$$
\alpha \sum_{x \in \mathfrak{I}}\left(\frac{1}{2} \log \frac{1}{2 I(x)}-\left(\frac{1}{2}-I(x)\right)\right)+\beta \sum_{x \in \mathfrak{I}}\left(I_{0}(x) \log \frac{I_{0}(x)}{I(x)}-\left(I_{0}(x)-I(x)\right)\right),
$$

where $\alpha, \beta>0$, which is based on the relative entropy distance 11 between $I$ and $1 / 2$ (the first term) and between $I_{0}$ and $I$ (the second term). Notice that, if $a>0$ and $f(s)=a \log \frac{a}{s}-(a-s), s \in(0,1]$, has a global minimum in $s=a$. In particular, this holds when $a=I_{0}(x)$ or $a=1 / 2$. Given the statistical interpretation of entropy, we can say that minimizing $D_{\alpha, \beta}^{\mathcal{E}}(I)$ amounts to minimizing the disorder of intensity levels around $1 / 2$ and around the original data $I_{0}(x)$. Thus, $D_{\alpha, \beta}^{\mathcal{E}}(I)$ accomplishes the required tasks of a dispersion term.

\section{Minimization of the Energy Functionals $E_{w, \alpha, \beta}^{f}(I)=D_{\alpha, \beta}^{\mathcal{E}}(I)+C_{w}^{f}(I)$}

The minimization of $E_{w, \alpha, \beta}^{f}(I), f=\mathrm{id}, \log ,-\mathcal{M}$ corresponds to a trade-off between two opposing mechanisms: on one hand we have entropic control of dispersion around $1 / 2$ and around original data, on the other hand we have local contrast enhancement.

The existence of a minimum in the discrete framework can be guaranteed for a quite general class of energy functionals see [1] for details. 
Assume that $\alpha, \beta>0$ are fixed. If $E_{w, \alpha, \beta}^{f}(I)=D_{\alpha, \beta}^{\mathcal{E}}(I)+C_{w}^{f}(I)$, then, by linearity of the variational derivative, we have $\delta E_{w, \alpha, \beta}^{f}(I)=\delta D_{\alpha, \beta}^{\mathcal{E}}(I)+\delta C_{w}^{f}(I)$. The minimum of $E_{w, \alpha, \beta}^{f}(I)$ satisfies $\delta E_{w, \alpha, \beta}^{f}(I)=0$. To search for the minimum a semi-implicit discrete gradient descent strategy with respect to $\log I$ can be used. This corresponds to using a gradient descent approach in which the metric is the relative entropy, instead of the usual quadratic distance (see [11]). The continuous gradient descent equation is

$$
\partial_{t} \log I=-\delta E_{w, \alpha, \beta}^{f}(I)
$$

with $t$ being the evolution parameter. Since $\partial_{t} \log I=\frac{1}{I} \partial_{t} I$, we have

$$
\partial_{t} I=-I \delta E_{w, \alpha, \beta}^{f}(I) .
$$

Let us now discretize the scheme: choosing a finite evolution step $\Delta t>0$ and setting $I^{k}(x)=I_{k \Delta t}(x), k \in \mathbb{N}$, with $I^{0}(x)$ being the original image being, then, by direct computation of $\delta D_{\alpha, \beta}^{\mathcal{E}}(I)$, the semi-implicit discretization of (11) is

$$
\frac{I^{k+1}(x)-I^{k}(x)}{\Delta t}=\alpha\left(\frac{1}{2}-I^{k+1}(x)\right)+\beta\left(I_{0}(x)-I^{k+1}(x)\right)-I^{k}(x) \delta C_{w}^{f}\left(I^{k}\right)(x) .
$$

The terms $-2 I^{k}(x) \delta C_{w}^{\text {id }}\left(I^{k}\right)(x) \equiv R_{w, I^{k}}^{\mathrm{id}}(x)$ and $-2 I^{k}(x) \delta C_{w}^{\log }\left(I^{k}\right)(x) \equiv R_{w, I^{k}}^{\log }(x)$ can be explicitly written as [1]:

$$
\begin{gathered}
R_{w, I^{k}}^{\mathrm{id}}(x)=\sum_{y \in \mathfrak{I}} w(x, y)\left[\frac{I^{k}(y)}{I^{k}(x)} \operatorname{sign}^{+}\left(I^{k}(x)-I^{k}(y)\right)-\frac{I^{k}(x)}{I^{k}(y)} \operatorname{sign}^{-}\left(I^{k}(x)-I^{k}(y)\right)\right] ; \\
R_{w, I^{k}}^{\log }(x)=\sum_{y \in \mathfrak{I}} w(x, y) \operatorname{sign}\left(I^{k}(x)-I^{k}(y)\right),
\end{gathered}
$$

where we set, for every $\xi \in \mathbb{R}$,

$\operatorname{sign}(\xi):=\left\{\begin{array}{l}1 \quad \text { if } \xi>0, \\ \frac{1}{2} \text { if } \xi=0, \\ -1 \text { if } \xi<0,\end{array} \quad \operatorname{sign}^{+}(\xi):=\left\{\begin{array}{l}1 \text { if } \xi>0, \\ \frac{1}{2} \text { if } \xi=0, \\ 0 \text { if } \xi<0,\end{array} \quad \operatorname{sign}^{-}(\xi)=1-\operatorname{sign}^{+}(\xi)\right.\right.$.

Equation (12) can be used to implement iterative computational algorithms for perceptual color image enhancement. In [12113] it is shown that the algorithm corresponding to $f=\log$ is a variational version of the ACE algorithm [14, while the one corresponding to $f=\mathrm{id}$ is a variational version of the (anti-)symmetrized Retinex algorithm 215. 


\section{An Image-Driven Contrast Enhancement Parameter}

From (12), it follows that the discrete equation that represents the variational method is the following:

$$
I^{k+1}(x)=\frac{I^{k}(x)+\Delta t\left(\frac{\alpha}{2}+\beta I^{0}(x)+\frac{1}{2} R_{I_{k}}^{f}(x)\right)}{1+\Delta t(\alpha+\beta)},
$$

$\alpha$ and $\beta$ represent the strength of the attachment to $1 / 2$ and to $I^{0}$, respectively. $R_{I_{k}}^{f}(x)$ performs contrast enhancement and can be written in the general form $R_{I_{k}}^{f}(x)=\sum_{y \in \mathfrak{I}} w(x, y) r^{f}\left(I^{k}(x), I^{k}(y)\right)$, where, for $f=\mathrm{id}, \log$ we have $r^{\mathrm{id}}\left(I^{k}(x), I^{k}(y)\right)=\frac{I^{k}(y)}{I^{k}(x)} \operatorname{sign}^{+}\left(I^{k}(x)-I^{k}(y)\right)-\frac{I^{k}(x)}{I^{k}(y)} \operatorname{sign}^{-}\left(I^{k}(x)-I^{k}(y)\right)$ and $r^{\log }\left(I^{k}(x), I^{k}(y)\right)=\operatorname{sign}\left(I^{k}(x)-I^{k}(y)\right)$.

In practice, the sign functions appearing in $r^{\text {id }}$ and $r^{\log }$ are too singular to be used without producing artifacts, so that a smoothed version is needed, we take $\operatorname{sign}_{s}\left(I^{k}(x)-I^{k}(y)\right)=\frac{\arctan \left(s\left(I^{k}(x)-I^{k}(y)\right)\right)}{\max _{y} \in \mathfrak{J}\left\{\left|\arctan \left(s\left(I^{k}(x)-I^{k}(y)\right)\right)\right|\right\}}, s>1$ defining its slope. It turns out that a proper election of the parameter $s$ is crucial to perform a suitable color enhancement.

So far, this parameter was let as a free user parameter, here we propose a formula to set $s$ as a function of image features in line with human visual perception, so that it does not need to be imposed by a user with an expensive try-and-look procedure.

The function we propose is the following

$$
s_{w}(x)=\frac{1}{\mu_{w}(x)} \cdot \frac{1-\sigma_{w}(x)}{\sigma_{w}(x)},
$$

where $\mu_{w}(x)$ is the local average intensity and $\sigma_{w}(x)$ is the local standard deviation $\sigma_{w}(x)$ :

$$
\mu_{w}(x)=\sum_{y \in \mathfrak{I}} w(x, y) I^{k}(y), \quad \sigma_{w}(x)=\sqrt{\sum_{y \in \mathfrak{I}} w(x, y)\left(I^{k}(y)-\mu_{w}(x)\right)^{2}} .
$$

The first factor ('WF factor') is introduced in accordance with the WeberFechner law: the HVS is more sensitive to contrast variations in low intensity areas rather than in bright ones, coherently with this, the WF factor increases $s_{w}(x)$ in low intensity areas. The second factor ('homogeneity factor') expresses the fact that the HVS is more sensible to contrast variations in homogeneous zones than in detailed ones, thus, using the local standard deviation as a measure of detail inside a given image area, we have that $s_{w}(x)$ increases when gets small. Because of the dependence on $x$, it is impossible to depict $s_{w}(x)$, however, it is clear that both the factors appearing in the formula show a hyperbolically decreasing behavior as the local average intensity or local standard deviation increase. 

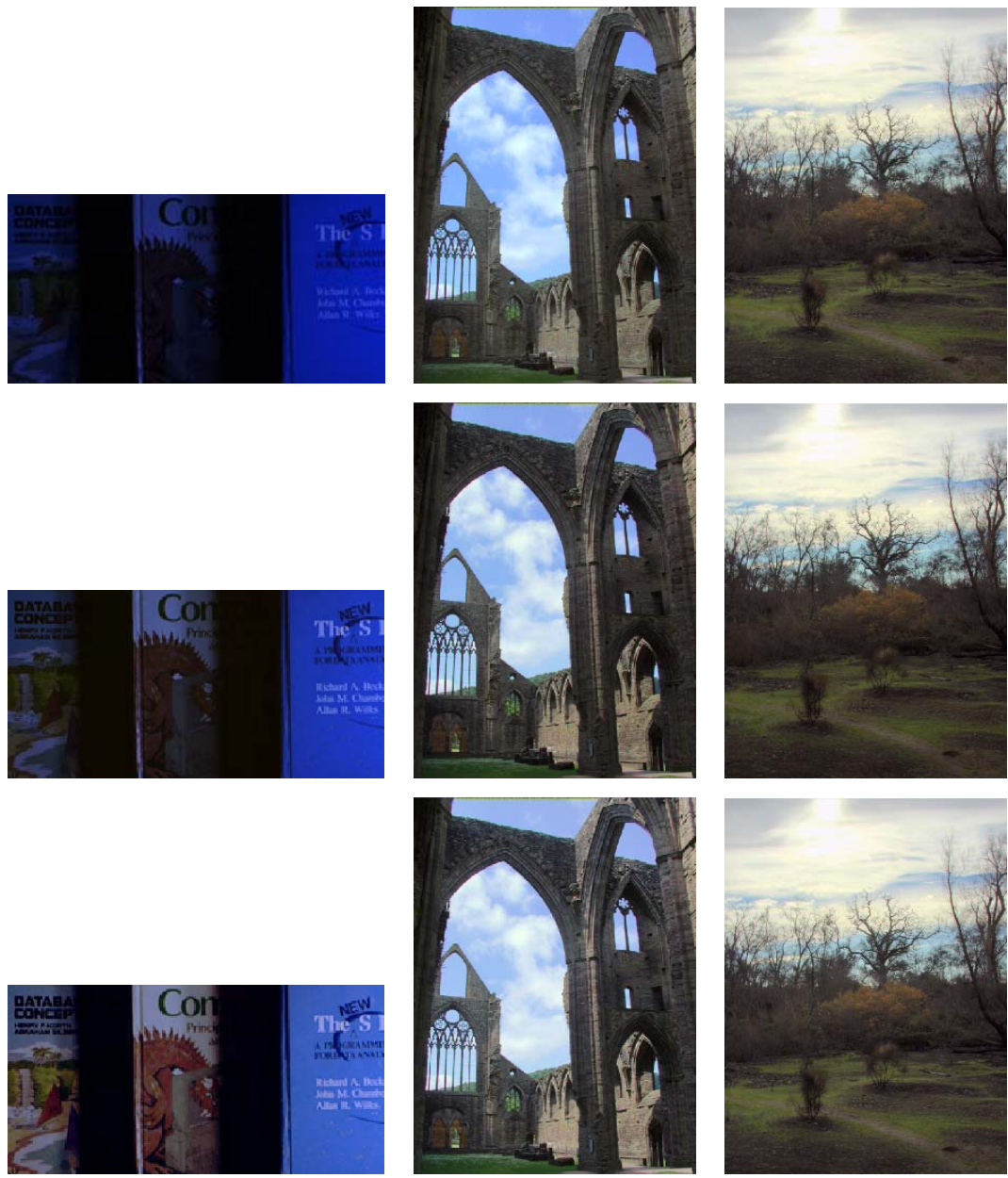

Fig. 1. First row: original images. Second row: filtered versions with fixed slope parameter $s=10$. Third row: filtered versions with varying slope parameter $s_{w}(x)$ as in (17). The contrast term used is $C_{w}^{\text {id }}$ and the parameters $\alpha$ and $\beta$ are both set to 1 .

Both $\mu_{w}(x)$ and $\sigma_{w}(x)$ can be computed through convolutions (denoted with the usual symbol $*$ ), which can be rapidly implemented thanks to the Fast Fourier Transform FFT. In fact, by definition, $\mu_{w}(x)=\left(I^{k} * w\right)(x)$ and, expanding the binomial expression in the definition of $\sigma_{w}(x)$,

$$
\sigma_{w}^{2}(x)=\sum_{y \in \mathfrak{I}} w(x, y) I^{k}(y)^{2}-2 \mu_{w}(x) \sum_{y \in \mathfrak{I}} w(x, y) I^{k}(y)+\mu_{w}(x)^{2} \sum_{y \in \mathfrak{I}} w(x, y)
$$

but, remembering that $w$ is normalized and the definition of $\mu_{w}(x)$ we can write $\sigma_{w}^{2}(x)=\left(w *\left(I^{k}\right)^{2}\right)(x)-\left[\left(w * I^{k}\right)(x)\right]^{2}$. 
Since the convolutions involved in the computation of $s(x)$ must be computed to approximate the function $R_{w, I^{k}}^{f}(x)$, as explained in [121], this formula does not increase the computational complexity of the algorithm, which remains $\mathcal{O}(N) \log (N)$, where $N$ is the total number of pixels.

Hereafter, we present some results that will show the soundness of this proposal for $s_{w}(x)$. The local automatic contrast parameter $s_{w}(x)$ still provides sound results and a better rendition of details in dark zones, as can be noticed in all images, in particular in the book one.

Remark: After many experiments, we empirically found out that a kernel function $w$ corresponding to overall good results is extended over the entire image and has this analytical expression $w(x, y)=1 /\|x-y\|_{\mathfrak{I}}$. It would be interesting to see if there is a relationship between this empirical function and physiological o psychophysical properties of human vision.

\section{Conclusions}

We have summarized a recently developed variational framework for perceptual color correction models [1. We have also proposed an image-driven formula to automatically set a parameter that influences the strenght of contrast enhancement. This function is in line with human vision properties and its computation does not increase the computational complexity of the algorithm.

\section{Acknowledgement}

The author acknowledges the Ramón y Cajal fellowship by Ministerio de Ciencia y Tecnología de España.

\section{References}

1. Palma-Amestoy, R., Provenzi, E., Caselles, V., Bertalmío, M.: A perceptually inspired variational framework for color enhancement. IEEE Transactions on Pattern Analysis and Machine Intelligence 31(3), 458-474 (2009)

2. Land, E., McCann, J.: Lightness and Retinex theory. Journal of the Optical Society of America 61(1), 1-11 (1971)

3. West, G.: Color perception and the limits of color constancy. Journal of Mathematical Biology 8, 47-53 (1979)

4. Hurlbert, A.: Formal connections between lightness algorithms. Journ. Opt. Soc. Am. A 3, 1684-1693 (1986)

5. Gonzales, R., Woods, R.: Digital image processing. Prentice-Hall, Englewood Cliffs (2002)

6. Creutzfeld, O., Lange-Malecki, B., Wortmann, K.: Darkness induction, retinex and cooperative mechanisms in vision. Exp. Brain Res. 67, 270-283 (1987)

7. Zaidi, Q.: Color and brightness induction: from Mach bands to three-dimensional configurations. Cambridge University Press, New York (1999) 
8. Shapley, R., Enroth-Cugell, C.: Visual adaptation and retinal gain controls 3, 263346 (1984)

9. Buchsbaum, G.: A spatial processor model for object colour perception. Journal of the Franklin Institute 310, 337-350 (1980)

10. Pratt, W.: Digital Image Processing. J. Wiley \& Sons, Chichester (2007)

11. Ambrosio, L., Gigli, N., Savaré, G.: Gradient flows in metric spaces and in the space of probability measures. Lectures in Mathematics. Birkhäuser, Basel (2005)

12. Bertalmío, M., Caselles, V., Provenzi, E., Rizzi, A.: Perceptual color correction through variational techniques. IEEE Trans. on Image Proc. 16, 1058-1072 (2007)

13. Bertalmío, M., Caselles, V., Provenzi, E.: Issues about Retinex Theory and Contrast Enhancement. International Journal of Computer Vision 83, 101-119 (2009)

14. Rizzi, A., Gatta, C., Marini, D.: A new algorithm for unsupervised global and local color correction. Pattern Recognition Letters 24, 1663-1677 (2003)

15. Provenzi, E., De Carli, L., Rizzi, A., Marini, D.: Mathematical definition and analysis of the Retinex algorithm. Journal of the Optical Society of America A 22(12), 2613-2621 (2005) 